\title{
When off label is off target
}

\author{
Off-label prescribing of drugs can add to a clinician's toolbox, but it may also pose risks to patients. Careful monitoring \\ and clinical validation should increase the safety and guarantee the efficacy of using existing drugs for new indications.
}

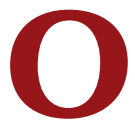

nce again, off-label prescribing has stirred controversy and concerns. The publication of three studies showing compelling evidence of health risks associated with off-label useand abuse in some hospitals—of the hemostatic agent recombinant factor VIIa (rFVIIa) raises questions as to how to properly regulate off-label drug use. The worrisome new findings call for a reassessment of the tools used by the US Food and Drug Administration (FDA) to examine the frequency of this practice among clinicians and the reasons encouraging doctors to prescribe off-label usage.

NovoSeven, the brand name of rFVIIa (Novo Nordisk), was approved to treat hemophiliacs during bleeding episodes or surgical procedures. But a post-marketing review of 35 studies showed increased risk of arterial thromboembolic events in patients with various clinical conditions, but not in healthy volunteers, treated with NovoSeven through off-label use (N. Engl. J. Med. 363 1791-1800, 2010). The FDA recently added a black-box warning to NovoSeven's label of serious thrombotic adverse events linked to off-label use, and some surgeons are now starting to be more cautious. Yet other physicians may still use this drug indiscriminately in light of the drug's potent, immediate effect at stopping bleeding in some patients, when other safer and cheaper hemostatics are available (http://www.nytimes.com/2011/04/19/health/19surgery.html).

Two more recent studies again raised the alarm about NovoSeven, underscoring a troubling trend: increased off-label use of drugs and lack of validation of efficacy and safety justifying off-label prescribing. Researchers at Stanford University retrospectively analyzed data from patients treated with rFVIIa between 2000 and 2008 in more than 600 hospitals in the US (Ann. Intern. Med. 154, 516-522, 2011). Strikingly, the use of rFVIIa for off-label indications increased 140-fold during that period, and by 2008 up to $97 \%$ of all prescriptions for rFVIIa were off label-a trend also observed for antipsychotics, allergy and asthma drugs and antibiotics - which raises the question of what clinical evidence has supported increased off-label prescribing over the last decade.

The same group showed that such evidence was lacking for rFVIIa. Randomized, controlled trials and observational studies comparing the use of rFVIIa with alternative therapies, placebo or standard care in hospitals failed to adequately show for five off-label indications that the drug was more or even as efficient at reducing mortality. For some indications, such as cardiac surgery, the drug in fact increased the risk of thromboembolism (Ann. Intern. Med. 154, 529-540, 2011).

There is no single scapegoat to blame for the public health consequences linked to off-label prescribing. Clinicians, patients, drug manufacturers and regulatory agencies all have a role. Off-label use permits clinicians to innovate and make potential discoveries with valuable implications for individuals with orphan diseases or who have failed prior therapies. And although off-label prescribing is legally permissible, health practitioners should recognize the limitations of case reports that attempt to demonstrate the safety and efficacy of drugs for unapproved indications. In addition, doctors could better inform their patients about whether the drugs prescribed are being used off label, the importance of reporting any adverse event and where and how to report a problem.

Off-label use can stem from empirical observations of potential drug applications, but drug companies often foster it. Direct marketing of drugs for off-label use is illegal, but manufacturers, under the right to commercial free speech, are allowed to share educational materials to teach clinicians and encourage them to improve clinical care with available drugs. The FDA released new guidelines last year to try to prevent biased dissemination of journal articles, but it still relies on the good faith of manufacturers. Regulatory agencies and hospital boards could have a more active role in supervising off-label use and the distribution of clinical journal articles.

The FDA also has a role in postmarketing survelliance. It uses a passive mechanism, the Adverse Event Reporting System, which allows for reporting through the MedWatch program. Patients and health practitioners can voluntarily report any adverse event, but companies are obliged to do so. When an adverse event is highly reported, the agency can impose a Risk Evaluation and Mitigation Strategy on the company to inform doctors and patients about these risks and limit the off-label use. But this may not be enough, as failure to submit a follow-up report and the lack of sufficient information are common problems. The FDA is now building an active surveillance system, the Sentinel Initiative, for monitoring drugs using electronic data from various healthcare data holders. This system may enable not only real-time tracking of the safety of approved drugs but also detection of adverse events linked to off-label use. This information will be analyzed by the FDA and disseminated to the public through the MedWatch program and the FDA's website. The Sentinel Initiative combined with further monitoring of educational activities may open a new era where doctors will have unbiased and timely evidence regarding off-label prescribing at hand to better execute therapy decisions.

Better regulation and formal testing would increase the clinical benefit and limit the risks of this useful practice. The off-label use of Avastin for macular degeneration is an encouraging example. It eventually led to a comparative clinical trial with Lucentis, its more expensive counterpart approved for the treatment of this disease, in which Avastin was shown to be similarly effective and safe. The off-label use of drugs can be used to give patients a more affordable and equally efficacious drug, but the risks should never outweigh the benefits. 